\title{
Transparent Monitoring of Rise-Time Using Asynchronous Amplitude Histograms in Optical Transmission Systems
}

\author{
Hui Chen, Student Member, IEEE, Student Member, OSA, Andrew W. Poon, Member, IEEE, Member, OSA, \\ and Xi-Ren Cao, Fellow, IEEE
}

\begin{abstract}
In this paper we report our analysis, simulations, and proof-of-principle experiment on transparent monitoring of relative rise-time using asynchronously sampled amplitude histograms in optical transmission systems. Our method is based on the count in a selected amplitude window between the mark and space levels. We evaluate our method under various amplitude windows and $Q$-factor values and compare with synchronously sampled eye-diagram based measurements. Our results demonstrate that the $40 \%-60 \%$ amplitude window is generally suitable for system rise-time monitoring purpose without clock information.
\end{abstract}

Index Terms-Amplitude histogram, asynchronous sampling, average $\mathbf{Q}$ factor, chromatic dispersion, performance monitoring.

\section{INTRODUCTION}

$\mathbf{P}$ ERFORMANCE monitoring is one of the key issues for modern optical transmission systems [1]. For digital communication channels, the ultimate performance criterion is given as bit-error-rate (BER). In amplitude modulation, BER is sensitive to both signal-to-noise ratio (SNR) and rise-time. While SNR is degraded by various types of noise effects, risetime is degraded by dispersion including chromatic dispersion (CD) and polarization-mode dispersion (PMD). Although various techniques have been proposed for transparently monitoring CD and PMD in optical transmission systems, including subcarrier monitoring [2], bit-rate frequency RF power monitoring [3], and vestigial-sideband optical filtering [4], these methods cannot provide a direct measurement on the system rise-time.

In this paper, we propose and demonstrate a method to monitor the transmission system rise-time using asynchronous amplitude histograms. Because asynchronous histograms require no clock information, the technique can be bit rate transparent. Asynchronous amplitude histograms can be efficiently obtained by optical sampling techniques [5] or by using an electroabsorption (EA) modulator [6]. It has been experimentally demonstrated that asynchronous histograms can

Manuscript received August 29, 2003. The work was supported by a Research Grants Council grant from Hong Kong University Grants Council. The work of A. W. Poon was partially supported by a grant from the University Grants Council of the Hong Kong Special Administrative Region, China. (Project No. HIA01/02.EG05).

The authors are with the Department of Electrical and Electronic Engineering, the Hong Kong University of Science and Technology, Clear Water Bay, Hong Kong Special Administrative Region, China. (email: chenh@ust.hk) be sensitive to the noise degradation, CD and fiber non-linear effects in optical transmission systems [7]. Shake et al. [8] and André et al. [9] recently suggested that the average Qfactor as measured from asynchronous histograms is closely related to the Q-factor as measured from the system BER. Although the average Q-factor depends on both the dispersion effects and noise effects [10], measuring the average Qfactor alone is not sufficient to identify system degradation sources. Measuring system rise-time will complement the asynchronous histogram-based average Q-factor techniques for system degradation source identification.

In section II, we first present an analytical model for extracting system rise-time from asynchronous histograms. In section III, we numerically examine the rise-time measurement and demonstrate transparent monitoring of dispersion under various Q-factor values. In section IV, we present our proofof-principle experiment that demonstrates the rise-time measurement under various Q-factor values for selected amplitude windows.

\section{Analytical Model}

Conventional synchronous amplitude histogram is a representation of the amplitude distributions of the mark and space levels. A synchronous temporal window is selected to obtain the distributions representing the mark and space levels near an optimal decision time. Fig. 1(a) shows the synchronous temporal window (solid line box) at the widest eye opening. Fig. 1(b) shows the corresponding histogram. Based on the histogram, the Q-factor and the BER can be estimated [11]. The Q-factor can be estimated as follows

$$
Q=\frac{\mu_{1}-\mu_{0}}{\sigma_{0}+\sigma_{1}}
$$

where $\mu_{0}$ and $\mu_{1}$ are the mean of the space and mark levels, $\sigma_{0}$ and $\sigma_{1}$ are the standard deviation of the space and mark levels [12].

However, when the clock information is absent, the signal can only be asynchronously sampled with no optimal time window. The amplitude histogram is obtained by random sampling that spans the entire bit period. With a sufficient number of random samples, an asynchronous histogram can evenly represent the pulse amplitude distribution in a bit period.

Figs. 1(c) and 1(d) show the asynchronously sampled eyediagram and histogram obtained within the entire bit period. The main difference between the asynchronous and the 


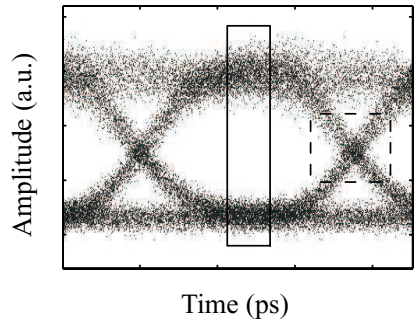

(a)

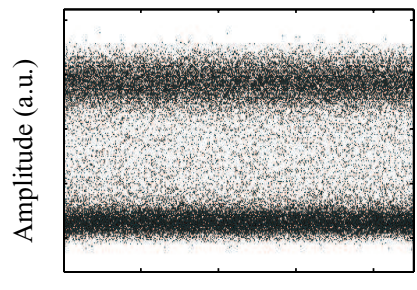

Time (ps)

(c)

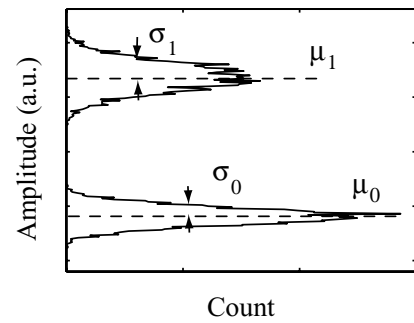

(b)

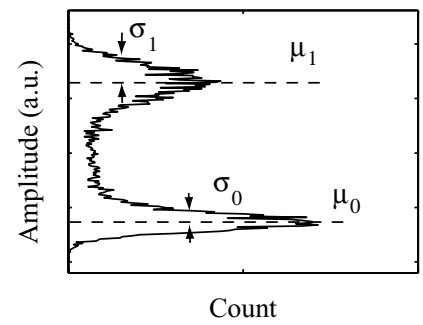

(d)
Fig. 1. (a) Eye-diagram and (b) histogram with synchronous sampling. (c) Eye-diagram and (d) histogram with asynchronous sampling.

synchronous histograms lies at the middle counts between the mark and space levels. We term the middle counts corresponding to the "cross" in the eye-diagram (dashed-line box in Fig. 1(a)) as "cross-point" counts. Within the crosspoint region, the asynchronous histogram (Fig. 1(d)) contains counts, while the synchronous histogram (Fig. 1(b)) contains essentially no counts.

Assuming the mark and space levels in asynchronous histograms have Gaussian distributions, we can obtain the Qfactor (known as average Q-factor [10]) based on (1). For the signal shown in Fig. 1, the average Q-factor is about 4.99 (Fig. 1(d)), while the Q-factor is about 5.78 (Fig. 1(b)). The average Q-factor is underestimated, because the $\sigma_{0}$ and $\sigma_{1}$ in asynchronous histograms are typically larger than those in synchronous histograms.

The cross-point count is related to the pulse rise-time and fall-time. For the following analysis, we consider as an example return-to-zero (RZ) pulses with Gaussian profiles. Fig. 2(a) shows two Gaussian-shaped RZ pulses having different pulse widths denoted as $A$ (solid line) and $B$ (dotted line). Within an arbitrarily chosen amplitude window $w$ between 0.4 and 0.6 normalized amplitude, the rise-time $\left(t_{w}\right)$ can be readily measured. Pulse $B$ has a longer rise-time than pulse $B$. Both the pulse rise edge and fall edge in the amplitude window contribute to the cross-point count in the asynchronous amplitude histogram. Since asynchronous histograms cannot distinguish the pulse rise time from the pulse fall time, we use the term "rise-time" to represent the average of the rise time and the fall time. Fig. 2(b) shows the asynchronous histograms of the pulse $A$ and $B$. Inset shows a zoom-in view of the selected amplitude window $w$. The histogram of pulse $B$ (dashed-line histogram) contains more cross-point counts than that of pulse $A$ (solid-line histogram). The comparison between Figs. 2(a) and 2(b) suggests that the cross-point count is approximately proportional to the pulse rise-time within a selected amplitude

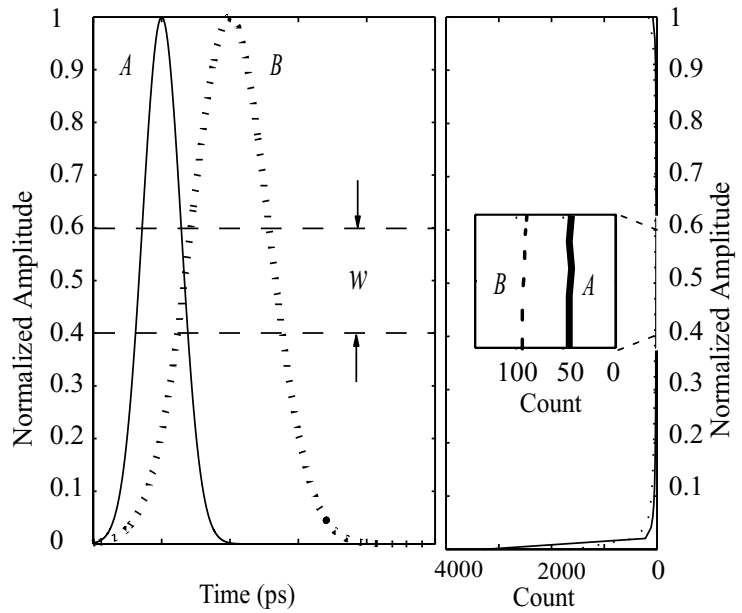

(a)

(b)

Fig. 2. (a) Calculated RZ pulses with different $10 \%-90 \%$ rise-times $(A$ : $0.085 \times$ bit period $B: 0.17 \times$ bit period). (b) The corresponding asynchronous amplitude histograms, solid line for pulse $A$ and dashed line for pulse $B$. Inset shows a magnified view of the histograms within the amplitude $w$.

window.

We measure the rise-time by using the cross-point count. We define the relative rise-time within an amplitude window $w$ as the rise-time to bit-period ratio $\left(t_{w} / T_{b}\right)$. Using the histogram total count $\left(C_{\text {total }}\right)$, we express the relative rise-time in terms of the cross-point count $\left(C_{w}\right)$ to the total count ratio. For a random bit sequence having an equal occurrence of marks and spaces in RZ format, we express the relative rise-time as,

$$
\frac{t_{w}}{T_{b}}=\frac{C_{w}}{C_{\text {total }}}
$$

For non-return-zero (NRZ) format, on average there occurs only one pulse edge in every two bit periods. We express the relative rise-time as,

$$
\frac{t_{w}}{T_{b}}=\frac{2 C_{w}}{C_{\text {total }}} .
$$

Assuming a linear pulse edge, we estimate for RZ format the $10 \%-90 \%$ pulse rise-time $T_{r}$ relative to $T_{b}$ as,

$$
\frac{T_{r}}{T_{b}}=\frac{0.8}{w} \frac{t_{w}}{T_{b}}=\frac{0.8}{w} \frac{C_{w}}{C_{\text {total }}} .
$$

Similarly for NRZ format, the $10 \%-90 \%$ pulse rise-time $T_{r}$ relative to $T_{b}$ can be given as [13],

$$
\frac{T_{r}}{T_{b}}=\frac{0.8}{w} \frac{t_{w}}{T_{b}}=\frac{0.8}{w} \frac{2 C_{w}}{C_{\text {total }}} .
$$

The relative rise-time measurement based on the cross-point count to total count ratio is bit-rate transparent. However, in order to measure the absolute rise-time $\left(T_{r}\right)$ using (4) and (5), a prior knowledge of the bit-period is required. For the following discussions, if not specify, we refer to the 10\%-90\% relative rise-time as the relative rise-time $\left(T_{r} / T_{b}\right)$.

NRZ and RZ formats have distinguishable asynchronous histograms. Figs. 3(a)-(d) show the simulated eye-diagrams and the corresponding asynchronous amplitude histograms for NRZ and RZ formats. We note that a moving average method 


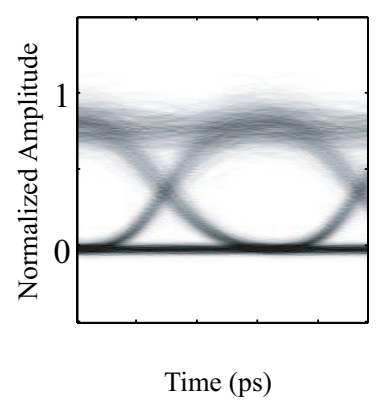

(a)

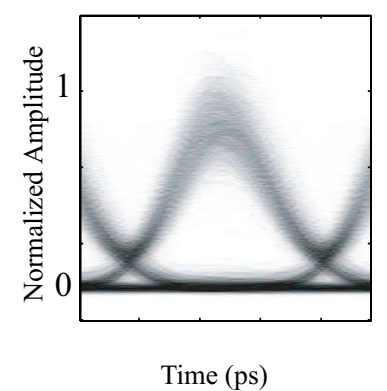

(c)

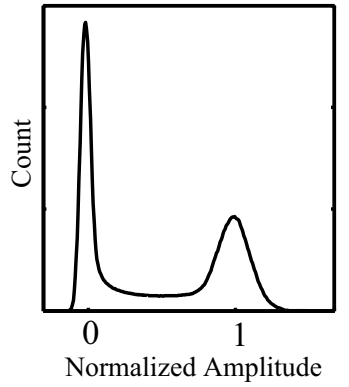

(b)

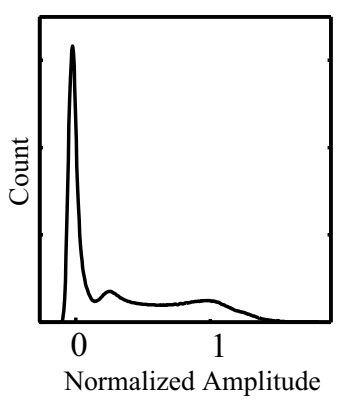

(d)
Fig. 3. (a) Eye-diagram and (b) asynchronous amplitude histogram of NRZ format. (c) Eye-diagram and (d) asynchronous amplitude histogram of RZ format.

is applied to smooth the histograms. The NRZ histogram (Fig. 3 (b)) contains two peaks. The RZ histogram (Fig. 3 (d)), only has one pronounced peak at the space level because the mark symbol only has a short duty cycle. The distinction between the NRZ and RZ asynchronous histograms enables the recognition of the NRZ and RZ formats without clock information. The relative rise-time can be obtained by (4) for RZ, and by (5) for NRZ.

\section{Simulation}

We numerically demonstrate the use of the cross-point count method to measure the system relative rise-time. Using simulations of dispersion degraded fiber-links, we analyzed rise-times obtained from asynchronous histograms and from eye-diagrams. We studied two issues that limit the histogrambased rise-time measurement accuracy and consistency: (i) under-estimation due to nonlinear pulse edges, and (ii) overestimation due to noise effects. These two issues impose contradicting requirements on choosing the amplitude window. We show that the $40 \%-60 \%$ amplitude window is suitable for dispersion degradation monitoring purpose under various noise levels and dispersion values .

\section{A. Simulation configuration}

We employed a commercially available communication systems design tool in our numerical simulations. Fig. 4 shows the schematic $40 \mathrm{~Gb} / \mathrm{s} 1550 \mathrm{~nm}$ transmission system. The fiber link was comprised of five loops of the same configuration. Each loop had a 50-km-long conventional single-mode fiber $(\mathrm{SMF})$ with a dispersion parameter $D_{S M F}=16 \mathrm{ps} /(\mathrm{nm} \cdot \mathrm{km})$,

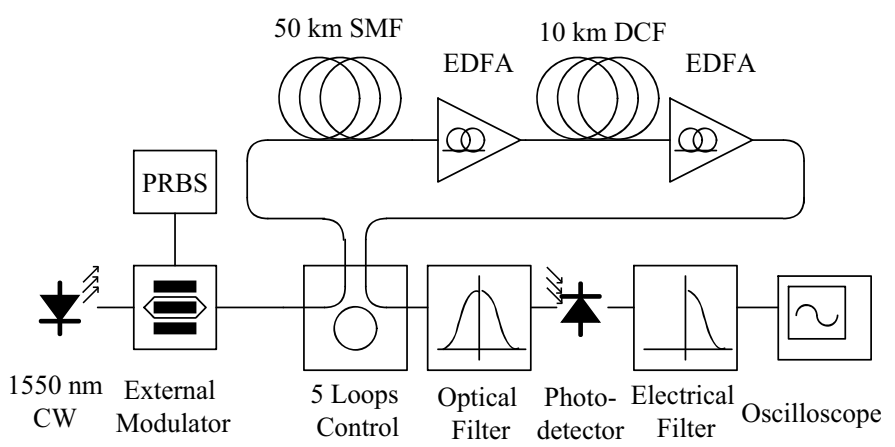

Fig. 4. Schematic of the simulation configuration. $D_{S M F}=16 \mathrm{ps} /(\mathrm{nm} \cdot \mathrm{km})$, $D_{D C F}=-80 \mathrm{ps} /(\mathrm{nm} \cdot \mathrm{km}), B_{\text {opt }}=160 \mathrm{GHz}, B_{\text {elec }}=30 \mathrm{GHz}$.

followed by a 10-km-long dispersion-compensation fiber (DCF) with a dispersion parameter $D_{D C F}=-80 \mathrm{ps} /(\mathrm{nm} \cdot \mathrm{km})$. The two erbium-doped fiber amplifiers (EDFAs) after each fiber loop compensate the fiber loss. The external modulator was driven by a $2^{16}-1$ pseudo-random bit sequence (PRBS) pattern. The optical filter bandwidth $B_{\text {opt }}$ was $160 \mathrm{GHz}$ and the electrical filter bandwidth $B_{\text {elec }}$ was $30 \mathrm{GHz}$. The system SNR was tuned by changing the photodetector noise. Eyediagrams and histograms were simulated.

\section{B. Relative rise-time calculations}

We tuned the $D_{S M F}$ in order to simulate different cumulative dispersion values through the fiber link. Fig. 5 shows the simulated NRZ asynchronous amplitude histograms with a cumulative dispersion value $D=0 \mathrm{ps} / \mathrm{nm}$ (solid line) and $D=64 \mathrm{ps} / \mathrm{nm}$ (dotted line), assuming a system SNR of approximately $21.6 \mathrm{~dB}$. Insets show the corresponding simulated eye-diagrams. Using the eye-diagrams, we obtained a $20 \%-80 \%$ rise-time of $8 \mathrm{ps}$ for the $D=0 \mathrm{ps} / \mathrm{nm}$ and of $10.75 \mathrm{ps}$ for the $D=64 \mathrm{ps} / \mathrm{nm}$. In the asynchronous amplitude histograms, the cross-point count has an observable increase with dispersion. Using the $20 \%-80 \%$ amplitude window and (3), we obtained a $20 \%-80 \%$ relative rise-time of 0.31 for the $D=0 \mathrm{ps} / \mathrm{nm}$ and of 0.43 for the $D=64 \mathrm{ps} / \mathrm{nm}$. These values correspond to a $20 \%-80 \%$ rise-time of 7.75 ps and of 10.75 ps in a $40 \mathrm{~Gb} / \mathrm{s}$ system. The difference between the histogrambased and the eye-diagram-based rise-time is below $3 \%$.

\section{Under-estimation due to nonlinear pulse edges}

The non-linear pulse edge can result in under-estimation of the relative rise-time. In practice, especially for highly dispersive systems, pulse edges are nonlinear (see the $D=64 \mathrm{ps} / \mathrm{nm}$ eye-diagram in Fig. 5 inset). Our method of estimating the relative rise-time by linear scaling from a small portion of the pulse edge can result in under-estimation. For the $64 \mathrm{ps} / \mathrm{nm}$ histogram shown in Fig. 5, the 40\%-60\% and 20\%-80\% relative rise-times based on cross-point count method are 0.13 and 0.43 . Using linear scaling, we obtained the relative rise times of 0.52 and of 0.57 , corresponding to $13 \mathrm{ps}$ and $14.25 \mathrm{ps}$ in a $40 \mathrm{~Gb} / \mathrm{s}$ system. Compared with the eye-diagram-based rise-time of $15 \mathrm{ps}$, the histogram-based measurements have an under-estimation exceeding $13 \%$ for the $40 \%-60 \%$ window 


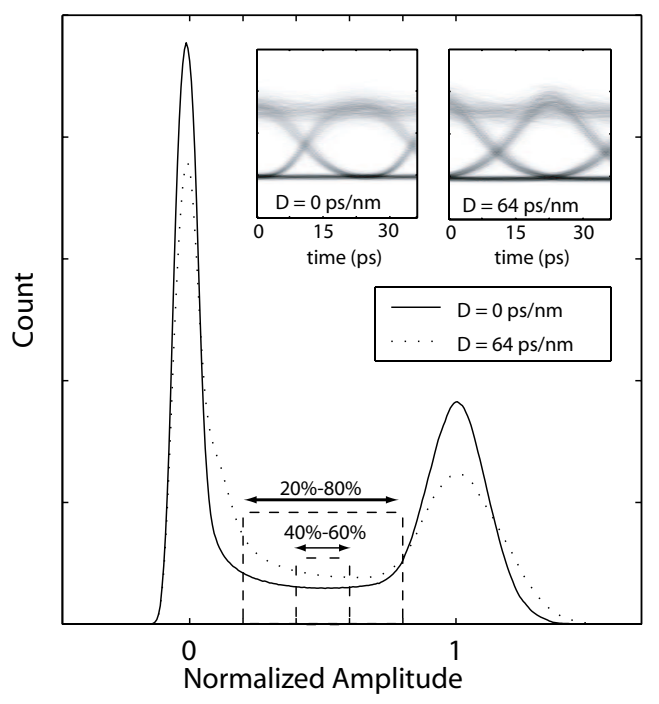

Fig. 5. Simulated NRZ asynchronous amplitude histograms for system $\mathrm{SNR} \approx 21.5 \mathrm{~dB}$. Cumulative dispersion of $D=0 \mathrm{ps} / \mathrm{nm}$ (solid line) and $D=64 \mathrm{ps} / \mathrm{nm}$ (dotted line). Insets show the corresponding eye-diagrams.

and below $5 \%$ for the $20 \%-80 \%$ window. In order to minimize such under-estimation, a wide amplitude window that includes the majority of the pulse edge is desirable.

\section{Over-estimation due to noise effects}

However, when noise effects are taken into account, the relative rise-time can be over-estimated. Fig. 6 shows the simulated asynchronous amplitude histograms for $D=0 \mathrm{ps} / \mathrm{nm}$ under various SNR values. In asynchronous histograms, the Gaussian distributions of the mark and space levels are broadened as SNR degrades. In the selected 35\%-55\% amplitude window, the cross-point counts are similar for $\mathrm{SNR} \approx 21.5 \mathrm{~dB}$ (solid line) and $\mathrm{SNR} \approx 16.9 \mathrm{~dB}$ (dashed line). However, the cross-point count in $\mathrm{SNR} \approx 12.8 \mathrm{~dB}$ histogram (dotted line) is slightly higher than that in the $21.5 \mathrm{~dB}$ and $16.9 \mathrm{~dB}$ histograms. The extra counts due to noise effects can result in over-estimation of the relative rise-time. Using simulated eye-diagrams, we measured the $35 \%-55 \%$ rise-time to be approximately $2.56 \mathrm{ps}$ for all the SNRs (with a higher uncertainty for the lower SNR). Using the histograms, we measured the rise-time to be about $2.5 \mathrm{ps}(21.5 \mathrm{~dB}), 2.48 \mathrm{ps}(16.9 \mathrm{~dB})$, and 2.8 ps $(12.8 \mathrm{~dB})$. The low $\mathrm{SNR} \approx 12.8 \mathrm{~dB}$ histogram results in approximately $10 \%$ over-estimation as compared with the eye-diagram-based measurement.

We remark that the over-estimation of the relative rise-time depends on the amplitude window $w$. A wider $w$ tends to result in a more severe over-estimation. For the $25 \%$ to $65 \%$ amplitude window, even the $16.9-\mathrm{dB}$ histogram can cause an over-estimation of about $7 \%$ as compared with the eyediagram-based measurement. In order to avoid rise-time overestimation in noisy systems, a narrower amplitude window is preferred.

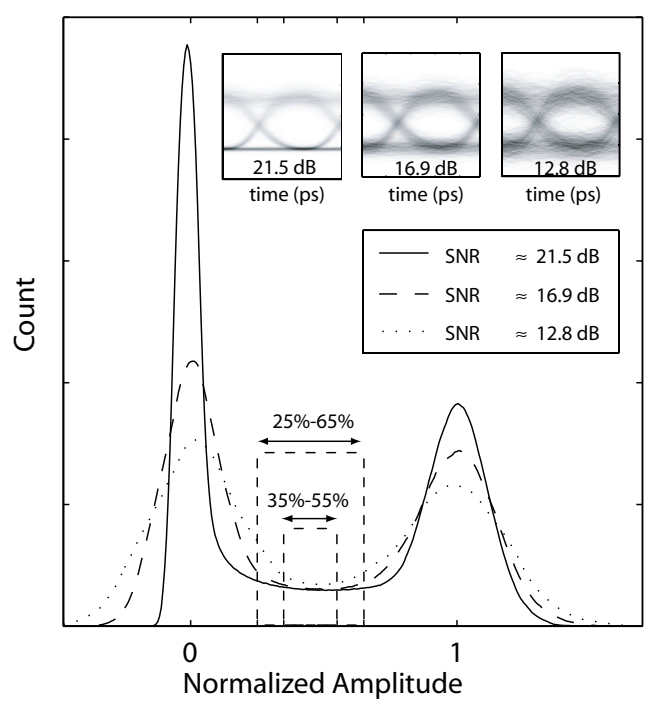

Fig. 6. Simulated NRZ asynchronous amplitude histograms for $D=0 \mathrm{ps} / \mathrm{nm}$. $\mathrm{SNR} \approx 21.5 \mathrm{~dB}$ (solid line), $\mathrm{SNR} \approx 16.9 \mathrm{~dB}$ (dashed line) and $\mathrm{SNR} \approx 12.8 \mathrm{~dB}$ (dotted line). Insets show the corresponding eye-diagrams.

\section{E. Choice of amplitude window}

Based on our simulations result, we found that the $40 \%$ $60 \%$ amplitude window is an optimal choice. Fig. 7 shows the calculated relative rise-time with the 40\%-60\% amplitude window under a scope of dispersion and noise values. The dotted lines show the relative rise-times measured from $\mathrm{Q} \approx 6$ eye-diagrams with the $40 \%-60 \%$ amplitude window under various dispersion values. For $\mathrm{Q}>4$, the histogram-based rise-times are consistently approximately $4 \%$ below the eyediagram-based rise-times. The histogram-based relative risetime variations between $\mathrm{Q}=3$ and $\mathrm{Q}=6$ remains below $5 \%$.

A wider $20 \%-80 \%$ amplitude window tends to cause significant system rise-time over-estimation in low-Q systems. Fig. 8 shows the relative rise-times with the $20 \%-80 \%$ amplitude window under a scope of dispersion and noise values. The dotted lines show the relative rise-times measured from $\mathrm{Q} \approx 6$ eye-diagrams with the $20 \%-80 \%$ amplitude window under various dispersion values. The relative rise-time overestimation in such a large window size is significant $(>10 \%)$ for low-Q systems $(\mathrm{Q}<4)$. However, when $\mathrm{Q} \geq 5$, the histogram-based relative rise-times have a better agreement with the eye-diagram-based calculations than those obtained from the $40 \%-60 \%$ amplitude window.

The general criterion of choosing the amplitude window is to cover the pulse edge that best represents the overall slope while keeping the window size adequately small to avoid noise effects. For rise-time monitoring purpose, the 40\%-60\% amplitude window is generally adequate.

\section{EXPERIMENT}

\section{A. Experimental setup}

Fig. 9(a) shows the schematic of our experimental setup. We used a wavelength tunable distributed feedback (DFB) laser in the $1550 \mathrm{~nm}$ wavelength range as the light source. 


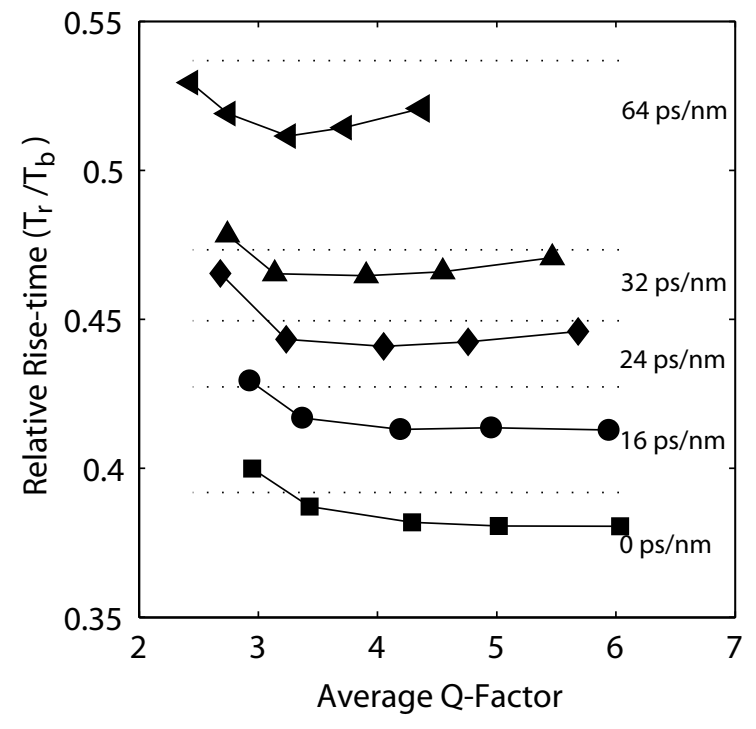

Fig. 7. Calculated relative rise-times (symbols) based on asynchronous amplitude histograms with the $40 \%-60 \%$ amplitude window. The solid lines are visual aids. The dotted lines show the measured relative rise-times from eye-diagrams with $\mathrm{Q} \approx 6$ under various dispersion values.

The laser light was fiber-coupled to a polarization controller. The linearly polarized light was amplitude modulated by a $10 \mathrm{~Gb} / \mathrm{s} \mathrm{LiNbO}_{3}$ Mach-Zehnder modulator driven by a $10 \mathrm{~Gb} / \mathrm{s}$ modulator driver. The bit stream was supplied by a bit-error-ratio tester (BERT) operating at $3.6 \mathrm{~Gb} / \mathrm{s} \mathrm{NRZ}$ format with a PRBS of $2^{32}-1$. The modulated optical signal was amplified by an erbium-doped fiber amplifier (EDFA) and coupled into a PMD emulator. The emulator provided a wavelength dependent differential group delay (DGD). We tuned the dispersion values by changing the laser wavelengths. The transmitted signal was detected by a $20 \mathrm{GHz}$ digital oscilloscope with a built-in photodetector. The system noise was adjusted by tuning the DFB laser output power. The asynchronous histograms were constructed without applying clock signals. The sampling number was approximately $7 \times$ $10^{7}$.

The PMD emulator was constructed by cascading three segments of polarization-maintaining (PM) fibers with the PM fiber principal axes purposely misaligned among the different segments. The PM fiber total length is about $40 \mathrm{~m}$. Our PMD emulator was designed to be fixed-length with a fixedorientation. We tested the PMD emulator by using standard fixed-analyzer method [14]. Fig. 9(b) shows the PMD emulator transmission after a fixed analyzer in the wavelength range between $1549.3 \mathrm{~nm}$ and $1550.9 \mathrm{~nm}$. Based on the maxima count over the wavelength span, we obtained the average DGD of about 45 ps. The period between the transmission maxima was about $0.15 \mathrm{~nm}$ to about $0.2 \mathrm{~nm}$.

\section{B. Relative rise-time measurements}

Fig. 10 shows the measured asynchronous histograms at two wavelengths, $1550.0 \mathrm{~nm}$ (solid line) and $1550.08 \mathrm{~nm}$ (dashed line), with an average $\mathrm{Q} \approx 8$. The $1550.0 \mathrm{~nm}$ histogram

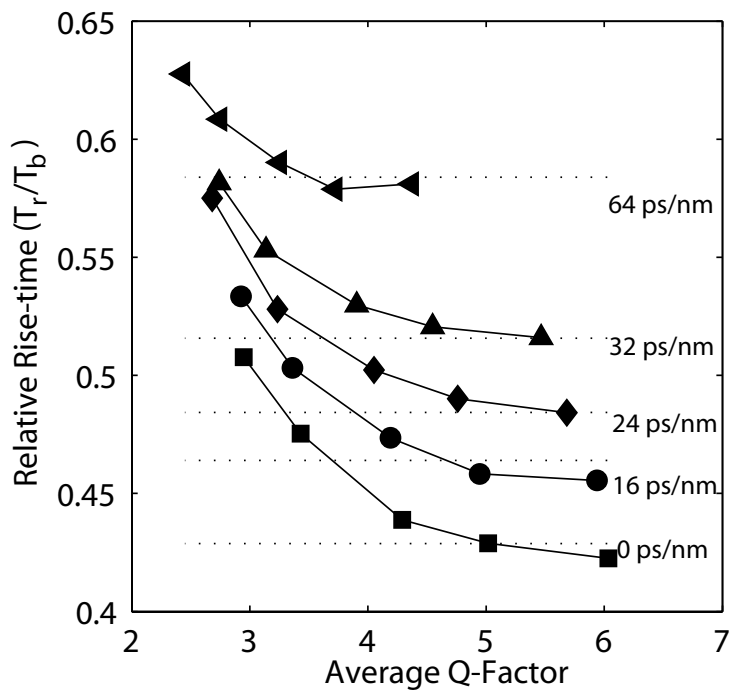

Fig. 8. Calculated relative rise-times (symbols) based on asynchronous amplitude histograms with the $20 \%-80 \%$ amplitude window. The solid lines are visual aids. The dotted lines show the measured relative rise-times from eye-diagrams with $\mathrm{Q} \approx 6$ under various dispersion values.

has larger cross-point count than the $1550.08 \mathrm{~nm}$ histogram. Using the 20\%-80\% amplitude window (dashed line) and (5), we obtained rise-time approximately as $94 \mathrm{ps}$ for $1550.0 \mathrm{~nm}$ and approximately as $60 \mathrm{ps}$ for $1550.08 \mathrm{~nm}$. Insets show the measured eye-diagrams. It is evident from the eye-diagrams that the $1550.0 \mathrm{~nm}$ signal has a rise-time of about $90 \mathrm{ps}$ the $1550.08 \mathrm{~nm}$ signal has a rise-time about $50 \mathrm{ps}$.

Fig. 11 shows the measured relative rise-times as a function of average Q-factor values at different wavelengths using the cross-point count within the 40\%-60\% amplitude window. When the average Q-factor is above 4, the measured rise-times at various $\mathrm{Q}$-factor values are consistent within $5 \%$. When the average Q-factor is below 4, the relative rise-time values increase slightly, which indicates a rise-time over-estimation due to noise effects.

Fig. 12 shows the measured relative rise-times as a function of average Q-factor values at different wavelengths using the cross-point count within the $25 \%-75 \%$ amplitude window. The over-estimation starts near the average $\mathrm{Q} \approx 6$. A $5 \%$ consistency can only be obtained for a large $Q-f a c t o r(Q \geq 6)$. The measured relative rise-times are slightly higher than those measured with the $40 \%-60 \%$ amplitude window (Fig. 11). This indicates an under-estimation due to non-linear pulse edges using a narrower amplitude window.

\section{PMD monitoring}

We used the measured relative rise-times to estimate the wavelength dependent DGD of the PMD emulator. The estimation was based on the approximated relation between the system rise-time and individual components rise-times given as [12],

$$
T_{r}^{2}=T_{t r}^{2}+T_{f i b e r}^{2}+T_{r e c}^{2}
$$




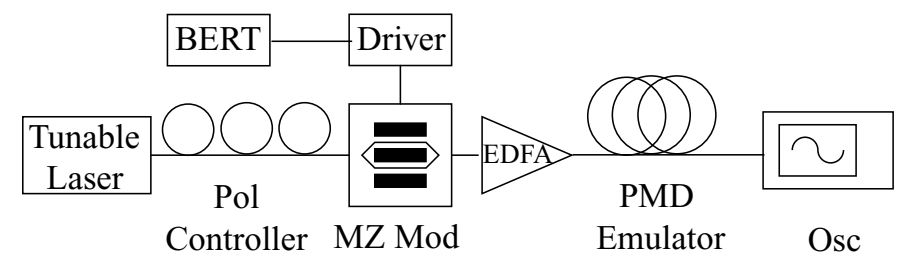

(a)

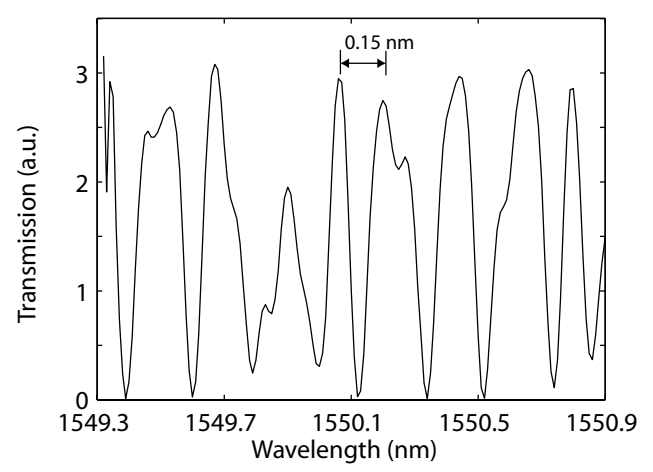

(b)

Fig. 9. (a) Schematic of the experimental setup. Pol Controller: Polarization controller; BERT: bit error ratio tester; Driver: Modulator driver; MZ Mod: $\mathrm{LiNbO}_{3}$ Mach-Zehnder modulator; OSC: High-speed oscilloscope with an photodetector. (b) Transmission spectrum of the PMD emulator using the fixed-analyzer method.

where $T_{t r}, T_{f i b e r}$ and $T_{r e c}$ are the rise-times for the transmitter, the fiber-link, and the receiver. In our experiment, these rise-times were dominated by the modulator driver rise-time $(\approx 48 \mathrm{ps})$, the PMD emulator DGD, and the oscilloscope rise-time $(\approx 25 \mathrm{ps})$, respectively. Fig. 13 shows the estimated DGD using (6) in the wavelength range between $1550.0 \mathrm{~nm}$ and $1550.2 \mathrm{~nm}$. The average DGD in this wavelength range was about $55 \mathrm{ps}$, which was consistent with the fixed-analyzer measurement of about 45 ps. The period between the DGD maxima $(\approx 0.14 \mathrm{~nm})$ and that between two DGD minima $(\approx 0.8 \mathrm{~nm})$ was also consistent with the period between the PMD emulator transmission maxima (Fig. 9(b)).

\section{CONClusion}

In summary, we propose and demonstrate an optical performance monitoring method based on asynchronous amplitude histograms to transparently monitor the system rise-time. This method uses the approximately linear relation between the cross-point count in an asynchronous amplitude histogram and the system rise-time. We studied the limitations of our proposed method, namely over-estimation due to noise effects and under-estimation due to non-linear pulse edges. Based on our numerical simulation results, we conclude that for a wide range of Q-factor values, the 40\%-60\% amplitude window is generally sufficient to provide an accurate relative risetime measurement within a 5\% consistency as compared with eye-diagram-based measurements. In our proof-of-principle experiment, we demonstrated the feasibility of monitoring PMD induced rise-time variations by using the 40\%-60\% amplitude window. We envision that the proposed method can be important for clock-less inline chromatic dispersion monitoring with active dispersion compensation units.

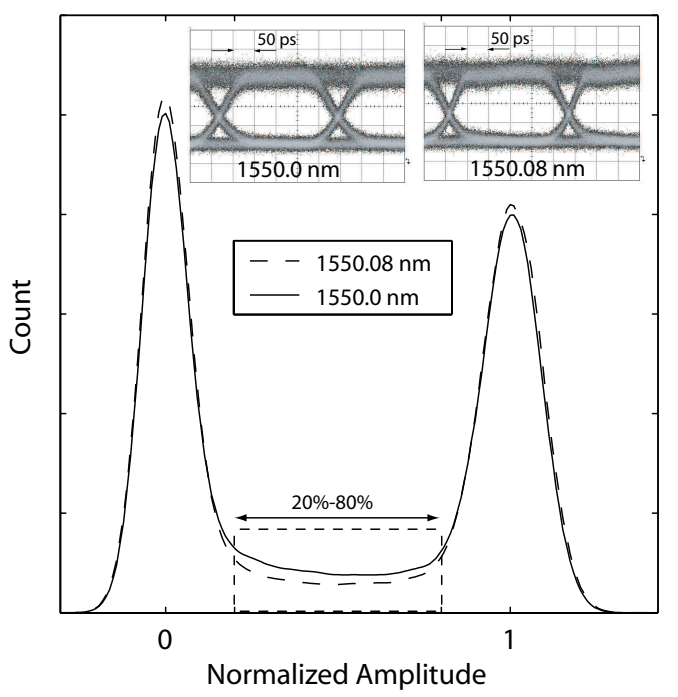

Fig. 10. Measured asynchronous amplitude histogram in wavelength 1550.0 $\mathrm{nm}$ (solid line) and $1550.08 \mathrm{~nm}$ (dashed line). Average $\mathrm{Q} \approx 8$. Insets show the corresponding eye-diagrams.

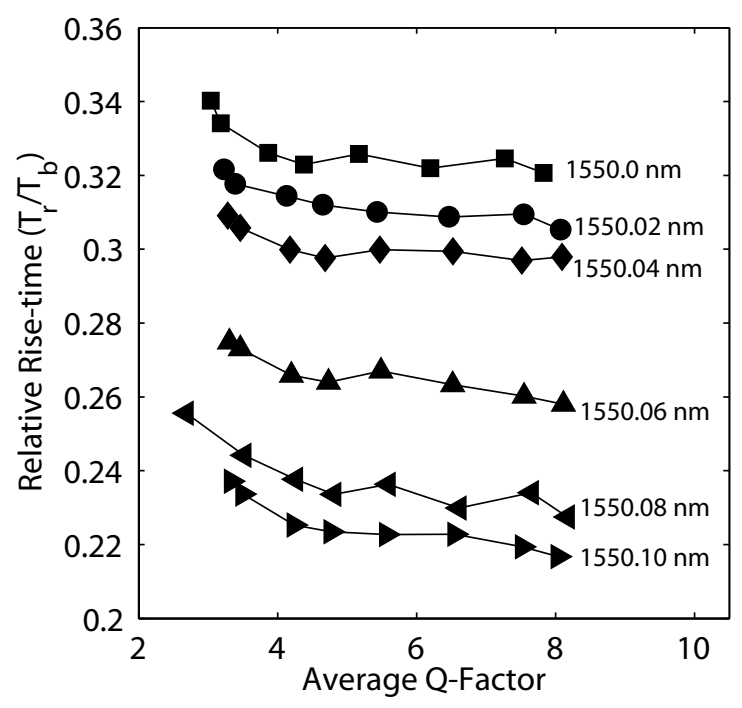

Fig. 11. Measured relative rise-times (symbols) at different wavelengths using asynchronous histograms with the $40 \%-60 \%$ amplitude window. The lines are visual aids.

\section{ACKNOWLEDGMENT}

The authors would like to thank Dr. Dennis T. K. Tong of HKUST for fruitful discussions. The authors would also like to thank Mr. S. L. Lee and Mr. K. W. Chan for their assistance on the experimental setup, and Dr. B. O. Guan of the Hong Kong Polytechnic University for the construction of the PMD emulator.

\section{REFERENCES}

[1] D. C. Kilper, R. Bach, D. J. Blumenthal, D. Einstein, T. Landolsi, L. Ostar, M. Preiss, and A. E. Willner, "Optical performance monitoring," $J$. Lightwave Technol., vol. 22, no. 1, pp. 294-304, Jan. 2004. 


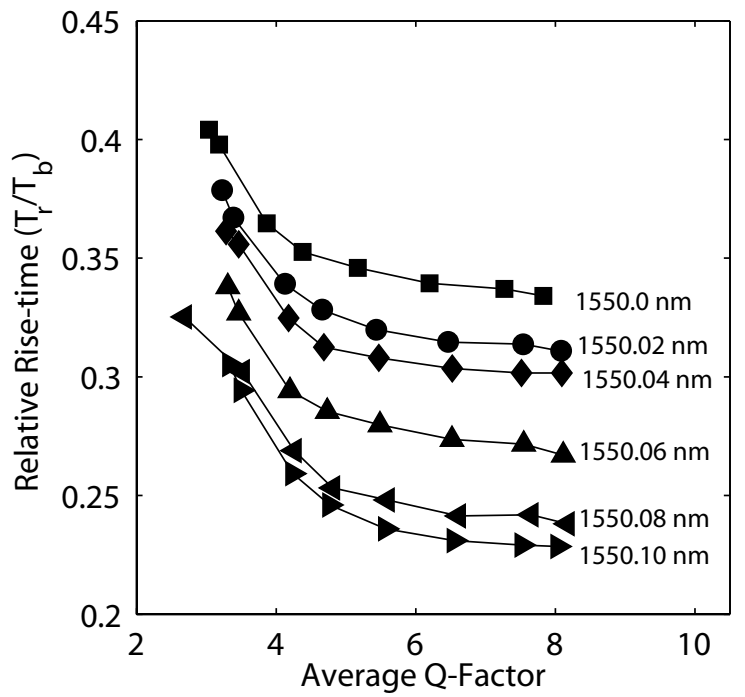

Fig. 12. Measured relative rise-times (symbols) at different wavelengths using asynchronous histograms with the $25 \%-75 \%$ amplitude window. The lines are visual aids.

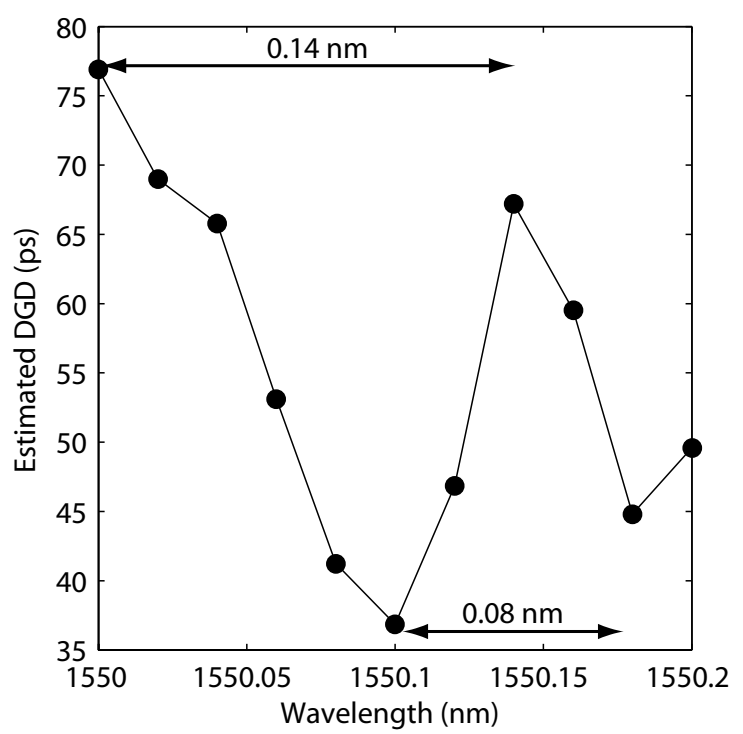

Fig. 13. Estimated DGD (symbols) from histogram-based measured risetimes. The line is a visual aid.

[2] G. Rossi, T. E. Dimmick, and D. J. Blumenthal, "Optical performance monitoring in reconfigurable WDM optical networks using subcarrier multiplexing," J. Lightwave Technol., vol. 18, no. 12, pp. 1639-1648, Dec. 2000.

[3] Z. Pan, Q. Yu, Y. Xie, S. A. Havstad, A. E. Willner, D. S. Starodubov, and J. Feiberg, "Chromatic dispersion monitoring and automated compensation for NRZ and RZ data using clock regeneration and fading without adding signal,' in Proc. OFC'2001, 2001, paper WH5-1.

[4] Q. Yu, L.-S. Yan, Z. Pan, and A. E. Willner, "Chromatic dispersion monitor for WDM systems using vestigial-sideband optical filtering," in Proc. OFC'02, 2002, paper WE3.

[5] H. Takara, S. Kawanishi, and M. Saruwatari, "Optical signal eye diagram measurement with subpicosecond resolution using optical sampling," Electron. Lett., vol. 32, no. 15, pp. 1399-1400, July 1996.

[6] I. Shake, H. Takara, and S. Kawanishi, "Simple Q factor monitoring for BER estimation using opened eye disgarms captured by high-speed asynchronous electrooptical sampling," IEEE Photon. Technol. Lett., vol. 15 , no. 4, pp. 620-622, Apr. 2003.
[7] K. Mueller, N. Hanik, A. Gladisch, H.-M. Foisel, and C. Caspar, "Application of amplitude histograms for quality of service measurements of optical channels and fault identification," in Proc. ECOC'98, 1998.

[8] I. Shake and H. Takara, "Averaged Q-factor method using amplitude histogram evaluation for transparent monitoring of optical signal-tonoise ratio degradation in optical transmission system," J. Lightwave Technol., vol. 20, no. 8, pp. 1367-1373, Aug. 2002.

[9] P. S. André, J. ao L. Pinto, A. L. J. Teixeira, J. F. da Rocha, T. Almeida, and M. Pousa, "Optical-signal-quality monitor for bit-error-ratio assessment in transparent dwdm networks based on asynchronously sampled amplitude histograms," Journal of Optical Netowrking, vol. 1, no. 3, pp. 118-128, Mar. 2002.

[10] I. Shake, H. Takara, K. Uchiyama, and Y. Yamabayashi, "Quality monitoring of optical signals influenced by chromatic dispersion in a transmission fiber using averaged q-factor evaluation," IEEE Photon. Technol. Lett., vol. 13, no. 4, pp. 385-387, Apr. 2001.

[11] N. S. Bergano, F. Kerfoot, and C. R. Davidson, "Margin measurements in optical amplifier systems," IEEE Photon. Technol. Lett., vol. 5, no. 3, pp. 304-306, 31993.

[12] G. P. Agrawal, Fiber-Optic Communication Systems, 2nd ed. New York: John Wiley \& Sons, 1997.

[13] H. Chen, A. W. Poon, and X.-R. Cao, "Amplitude histogram-based method for inline pulse rise time monitoring without eye-diagram," in Proc. CLEO'03, 2003, paper CThJ4.

[14] C. D. Poole and D. L. Favin, "Polarization-mode dispersion measurements based on transmission spectra trhough a polarizer," J. Lightwave Technol., vol. 12, no. 6, pp. 917-929, June 1994.

Hui Chen Biography text here.

Andrew W. Poon Biography text here.

Xi-Ren Cao Biography text here. 